\title{
Reduced mitochondrial density in the vastus lateralis muscle of patients
} with COPD

\author{
H.R. Gosker*, M.K.C. Hesselink" ${ }^{\#}$ H. Duimel ${ }^{*}$, K.A. Ward* and A.M.W.J. Schols*
}

ABSTRACT: Skeletal muscle dysfunction is a well-recognised hallmark of chronic obstructive pulmonary disease (COPD) leading to exercise intolerance. The vastus lateralis of COPD patients is characterised by reduced mitochondrial enzyme activity; however, this is not the case in the tibialis anterior. It is, however, unclear whether the compromised oxidative capacity in the vastus is due to reduced mitochondrial volume density.

Muscle biopsies were obtained from the vastus lateralis of six COPD patients and four healthy age-matched controls, and from the tibialis anterior of another six COPD patients and six controls. Mitochondrial number, fractional area and morphometry, as well as Z-line width (as a surrogate marker of fibre type), were analysed using transmission electron microscopy.

Mitochondrial number $\left(0.34\right.$ versus $\left.0.63 \mathrm{n} \cdot \mu^{-2}\right)$ and fractional area (1.95 versus $\left.4.25 \%\right)$ were reduced in the vastus of COPD patients compared with controls. Despite a reduced mitochondrial number $\left(0.65\right.$ versus $\left.0.88 \mathrm{n} \cdot \mu \mathrm{m}^{-2}\right)$, the mitochondrial fractional area was maintained in the tibialis of COPD patients compared with controls.

It can be concluded that the reduced mitochondrial fractional area is likely to contribute to the decreased oxidative capacity in the vastus of chronic obstructive pulmonary disease patients, whereas the maintained mitochondrial fractional area in the tibialis may explain the normal oxidative capacity.

KEYWORDS: Chronic obstructive pulmonary disease, electron microscopy, mitochondria, skeletal muscle, ultrastructure

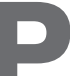
eripheral muscle dysfunction is an established hallmark of chronic obstructive pulmonary disease (COPD). It severely affects patients' exercise tolerance leading to disability and poor quality of life. Several underlying muscular metabolic derangements have been reported for the vastus lateralis muscles of patients with COPD, including a shift from oxidative type-I (slow-twitch) towards glycolytic type-II (fast-twitch) fibres [1, 2] and reduced activity of the enzymes involved in oxidative energy metabolism $[3,4]$. These enzymes, which are involved in the citric acid cycle and fatty acid $\beta$-oxidation, reside within the mitochondria. An increased mitochondrial fractional area has indeed been associated with an increased oxidative enzyme capacity in human skeletal muscle [5]. It can, therefore, be hypothesised that in COPD the reduced oxidative enzyme capacity in the vastus lateralis is the consequence of a reduced mitochondrial fractional area. In COPD, the ultrastructure of muscle mitochondria has, so far, only been studied in the diaphragm [6]. As opposed to the vastus lateralis, the diaphragm of these patients is characterised by an increased oxidative capacity [7-9] and, strikingly, an augmented mitochondrial density was found when compared with healthy age-matched controls [6]. Similarly, since normal oxidative enzyme activities have been reported for COPD in another peripheral muscle, the tibialis anterior [10], a normal mitochondrial fractional area is anticipated in this muscle.

Therefore, the aim of the current study was to examine muscle mitochondria on the ultrastructural level in the vastus lateralis or tibialis anterior muscle of patients with COPD as compared with healthy subjects. For this, muscle biopsies were obtained and mitochondrial number and fractional area along with mitochondrial morphometry were analysed using transmission electron microscopy. Since alterations in mitochondrial fractional area may result in

\section{AFFILIATIONS}

Depts of *Respiratory Medicine,

${ }^{\#}$ Movement Sciences, and

-Pathology, EM Unit, Nutrition and

Toxicology Research Institute

Maastricht (NUTRIM), Maastricht

University, Maastricht, The

Netherlands.

CORRESPONDENCE

H.R. Gosker

Dept of Respiratory Medicine

Maastricht University

Nutrition and Toxicology Research

Institute Maastricht

P.0. Box 616

6200 MD Maastricht

The Netherlands

Fax: 31433875051

E-mail: H.Gosker@pul.unimaas.nl

Received:

November 102006

Accepted after revision:

March 262007

\section{SUPPORT STATEMENT}

The research of H. R. Gasker was supported by an award from the Netherlands Asthma Foundation (No. 3.2.05.038)

STATEMENT OF INTEREST

None declared. 
disturbances in fuel stores, a secondary aim was to examine glycogen and lipid droplets at the ultrastructural level.

\section{METHODS}

A total of 12 patients with mild-to-severe airflow obstruction and 10 healthy control subjects were studied (table 1). A vastus lateralis biopsy was obtained from six patients and four controls. A biopsy from the tibialis anterior was obtained from another six patients and six controls. All patients had COPD as defined according to the Global Initiative for Chronic Obstructive Lung Disease criteria [11]. Furthermore, all patients had irreversible obstructive airway disease $(<10 \%$ improvement of forced expiratory volume in one second (FEV1) predicted baseline after $\beta_{2}$-agonist inhalation). They were in a clinically stable condition, did not suffer from a respiratory tract infection or an exacerbation of the disease at least 4 weeks prior to the study, and they were receiving one maintenance dose of inhaled steroids and $\beta_{2}$-agonist but did not receive systemic steroids. Exclusion criteria were: malignancy; asthma; bronchiectasis; cardiac failure; distal arteriopathy; recent surgery; severe endocrine, hepatic or renal disorders; and use of anticoagulant medication. The control subjects were healthy age-matched volunteers. Written informed consent was obtained from all subjects and the study was approved by the medical ethical committee of the University Hospital Maastricht (Maastricht, the Netherlands) consistent with the Helsinki Declaration.

\begin{tabular}{|c|c|c|c|c|}
\hline & \multicolumn{2}{|c|}{ Vastus lateralis biopsy } & \multicolumn{2}{|c|}{ Tibialis anterior biopsy } \\
\hline & Controls & COPD & Controls & COPD \\
\hline Subjects $n$ & 4 & 6 & 6 & 6 \\
\hline Male/female $n$ & $3 / 1$ & $4 / 2$ & $6 / 0$ & $6 / 0$ \\
\hline Age yrs & $60.5 \pm 3.1$ & $60.0 \pm 11.8$ & $65.2 \pm 6.6$ & $66.5 \pm 9.6$ \\
\hline Length $\mathrm{m}$ & $1.71 \pm 0.05$ & $1.69 \pm 0.06$ & $1.79 \pm 0.04$ & $1.75 \pm 0.06$ \\
\hline Weight kg & $80.8 \pm 8.3$ & $75.8 \pm 18.7$ & $84.3 \pm 5.0$ & $66.0 \pm 10.7^{\star}$ \\
\hline $\mathrm{BMI} \mathbf{k g} \cdot \mathrm{m}^{-2}$ & $27.5 \pm 1.6$ & $26.5 \pm 5.7$ & $26.3 \pm 2.0$ & $21.5 \pm 3.5^{\star}$ \\
\hline FFM kg & $61.0 \pm 8.6$ & $53.5 \pm 6.8$ & $81.7 \pm 3.6$ & $53.5 \pm 6.0^{\#}$ \\
\hline $\mathrm{FFMI} \mathbf{k g} \cdot \mathrm{m}^{-2}$ & $20.7 \pm 2.0$ & $18.3 \pm 2.5$ & $25.5 \pm 1.3$ & $17.6 \pm 3.0^{\#}$ \\
\hline FEV $1 \%$ pred & $99.5 \pm 11.4$ & $53.7 \pm 22.9$ * & $111.7 \pm 10.6$ & $35.4 \pm 11.6^{*}$ \\
\hline FVC $\%$ pred & $109.3 \pm 13.3$ & $88.7 \pm 21.3$ & $117.3 \pm 2.0$ & $84.6 \pm 19.5^{\star}$ \\
\hline $\mathrm{FEV}_{1 / \mathrm{FVC}} \%$ & $73.3 \pm 0.9$ & $47.7 \pm 13.8^{*}$ & $73.0 \pm 8.9$ & $32.8 \pm 8.7^{\#}$ \\
\hline DL,Co \% pred & $114.0 \pm 11.7$ & $86.1 \pm 19.3^{*}$ & $123.4 \pm 20.8$ & $65.8 \pm 39.6^{*}$ \\
\hline \multicolumn{5}{|l|}{ Smoking habits } \\
\hline Smoker & 0 & 0 & 1 & 1 \\
\hline Nonsmoker & 0 & 0 & 2 & 1 \\
\hline Ex-smoker & 4 & 6 & 3 & 4 \\
\hline
\end{tabular}

Data are presented as mean $\pm \mathrm{SD}$, unless otherwise stated. COPD: chronic obstructive pulmonary disease; BMI: body mass index; FFM: fat-free mass; FFMI: FFM index; FEV1: forced expiratory volume in one second; \% pred: \% predicted; FVC: forced vital capacity; $\mathrm{LL}, \mathrm{CO}$ : diffusing capacity of the lung for carbon monoxide. ${ }^{*}: p<0.05$ compared with controls; ${ }^{*}: p \leqslant 0.01$ compared with controls.

\section{Body composition}

Body height and weight were assessed. Whole body fat-free mass (FFM) was determined by bioelectrical impedance (Xitron 4000b; Xitron technologies, San Diego, CA, USA) as described previously elsewhere [12]. Weight parameters were adjusted for body surface, resulting in the body mass index and FFM index.

\section{Pulmonary function tests}

Spirometry was used to determine, amongst others, FEV1, with the highest value from at least three technically acceptable assessments being used. The diffusing capacity of the lung for carbon monoxide was measured using the single-breath method (Masterlab; Jaeger, Wurzburg, Germany). All values obtained were expressed as percentage of the predicted value [13].

\section{Muscle biopsy and electron microscopic evaluation}

Post-absorptive muscle biopsies were obtained under local anaesthetic under resting conditions. Vastus lateralis biopsies were obtained using the needle biopsy technique [14]. Tibialis anterior biopsies were obtained using a conchotome [15]. The specimens were immediately fixed in $0.1 \mathrm{M}$ phosphate buffer with $2.5 \%$ glutaraldehyde at $\mathrm{pH} 7.4$ and stored in a dark, cool place in the fixation buffer. For electron microscopy, specimens were rinsed in $0.1 \mathrm{M}$ phosphate buffer and post-fixed in the fixation buffer supplemented with $1 \%$ osmiumtetroxide for $1 \mathrm{~h}$, dehydrated through a graded ethanol series and embedded in epoxy resin (Glycid ether 100; Serva, Heidelberg, Germany). Appropriate locations and fibre longitudinal orientation were evaluated in toluidine blue-stained semi-thin sections from the central region of each biopsy. Ultra-thin sections from the selected areas were contrasted with uranyl acetate and lead citrate and viewed with a Philips CM 100 transmission electron microscope (Philips, Eindhoven, the Netherlands). Using this staining protocol, mitochondria will appear as spherical round-shaped electron dense (dark grey or black) objects. Mitochondrial analysis was based on the basic standards described previously elsewhere [5, 16, 17]. Micrographs of randomly selected areas of central parts of muscle fibres were obtained at a final magnification of 1,550 $2,650 \times$ and scanned at $>2,400 \mathrm{dpi}$. Mitochondria were identified based on their electron dense (dark grey or black) appearance, shape and subcellular location (intermyofibrillar). A representative micrograph is shown in figure 1a. These digitised micrographs were analysed with an interactive image analysis system (QWin v2.8; Leica Microsystems Imaging Solutions, Cambridge, UK). All mitochondria entirely within the micrograph were included, 3-5 micrographs were analysed so that at least 275 mitochondria per subject were examined. Mitochondria were outlined in an overlaying bitmap (fig. 1b), which was used to compute (tables 2 and 3) least diameter, perimeter and size (area). Subsequently, mitochondrial number was computed as the quantity of mitochondria in a given muscle fibre area. Mitochondrial fractional area was computed as the percentile mitochondrial area fraction of total fibre area. As a surrogate marker of muscle fibre typology [18], the average Z-line width was also determined for each micrograph: 10 randomly chosen straight Z-lines were outlined in an overlaying bitmap and the mean width of these objects was measured. Furthermore, glycogen and lipid stores were evaluated semi-quantitatively in a 
blinded manner. The entire section was evaluated under the electron microscope for a general impression and then quantity scores (1-5) were assigned using the same digitised micrographs aforementioned. For each subject the scores from these micrographs were averaged.

\section{Statistics}

Differences between groups were analysed with the MannWhitney U-test and correlations with the Spearman correlation test. A two-tailed probability value $<0.05$ was considered statistically significant. Data are presented as mean \pm SD.

\section{RESULTS}

The characteristics of patients and healthy controls are presented in table 1. Age and anthropometric parameters were not different between the two groups. In patients, the predicted value for FEV1 varied between 22 and $84 \%$ and the FEV1/forced vital capacity (FVC) ratio varied from 25 to $68 \%$.

Electron microscopic analysis of mitochondria is presented in tables 2 and 3 (vastus lateralis biopsies and tibialis biopsies, respectively). For both muscles, mean Z-line width, which is an indicator of fibre type, was similar between patients and controls, indicating that muscle fibre typology of the samples under examination was comparable and that the data shown are not biased by differences in muscle fibre typology. In the vastus lateralis, determinants of mitochondrial size (mitochondrial area, least diameter and perimeter) were also not different between the two groups, although there was one subject in the control group (control 1) with larger mitochondria compared with the other subjects. Mitochondrial number, as well as fractional area, was lower in vastus lateralis muscle samples from COPD patients than from controls. Even when control 1 was omitted from the analysis, mitochondrial fractional area was still significantly lower in patients $(\mathrm{p}=0.039)$. Interestingly, mitochondrial number was also lower in tibialis samples of COPD patients compared with controls, whereas the mitochondrial fractional area remained unaltered. Mitochondrial morphometry (size, diameter and perimeter) revealed no significant differences between both groups, although mitochondrial size (area and least diameter) tended to be larger in the tibialis from COPD patients $(p=0.109)$ as compared with controls.

In COPD, there were no significant correlations between mitochondrial fractional area and disease severity expressed as either FEV1 (fig. 2) or FEV1/FVC (not shown). No significant correlations were observed between these disease severity measures and mitochondrial number or other mitochondrial dimensions (not shown).

Quantity scores for the presence of fuel stores are presented in tables 4 and 5. The evaluation of glycogen and lipid stores in the vastus lateralis or tibialis samples revealed no significant differences between patients and healthy subjects.

\section{DISCUSSION}

The main finding of the present study was the reduced mitochondrial number and fractional area in the vastus lateralis muscle of patients suffering from COPD compared with age-matched healthy controls. A reduced mitochondrial fractional area was, however, not observed in the tibialis anterior of COPD patients. This indicates that the reduced oxidative capacity in the vastus lateralis and the absence of this phenomenon in the tibialis anterior could very well be attributed to their respective mitochondrial fractional area.

In the present study, mitochondrial density was expressed either as the mitochondrial number or as the fractional area. The mitochondrial number is the amount of mitochondria located in a certain muscle fibre area, whereas the mitochondrial fractional area is the area fraction of mitochondria. In other words, a large number of small mitochondria can result in the same mitochondrial fractional area as a small number of large mitochondria. Furthermore, changes in mitochondrial
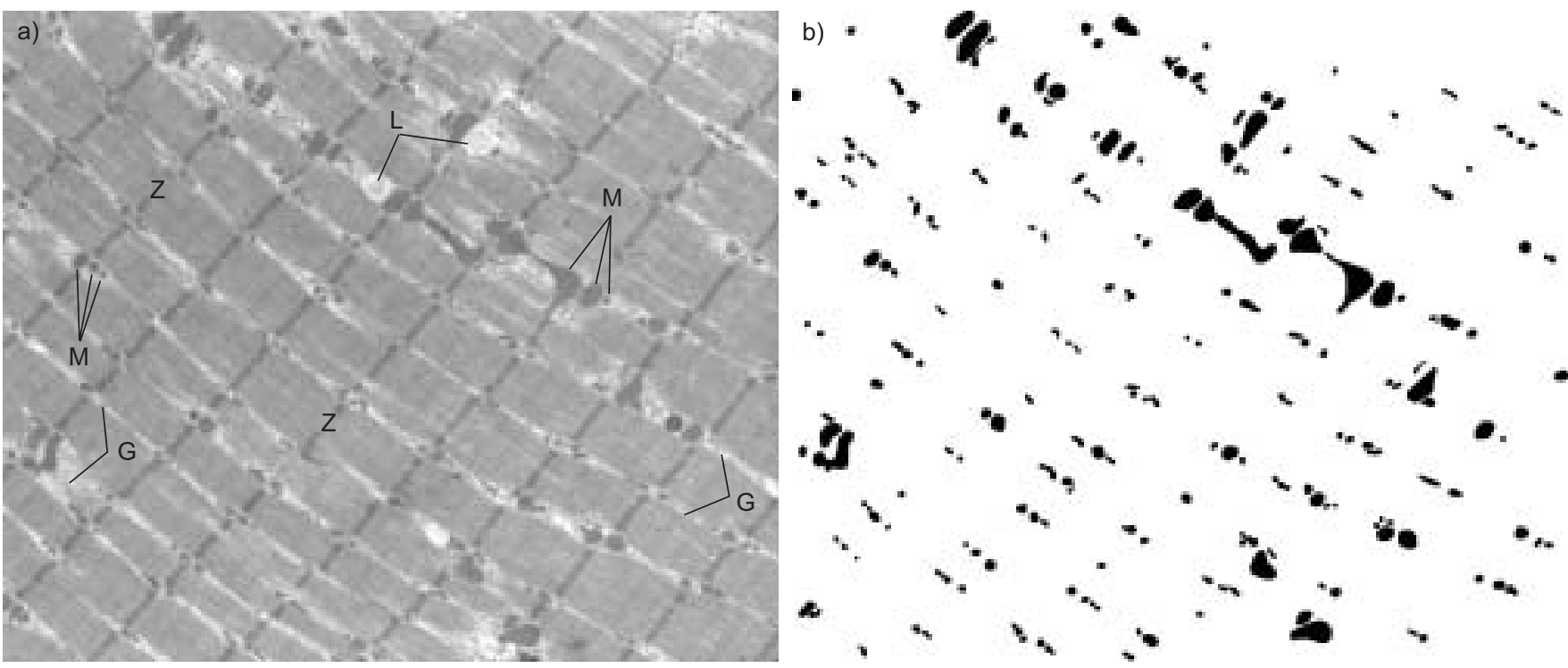

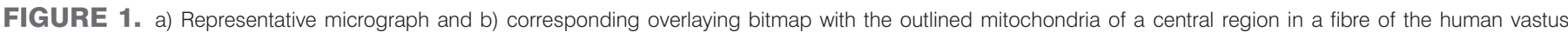

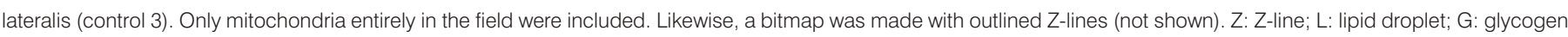
stores; M: mitochondria. 


\section{TABLE 2 Mitochondrial parameters in vastus lateralis biopsies}

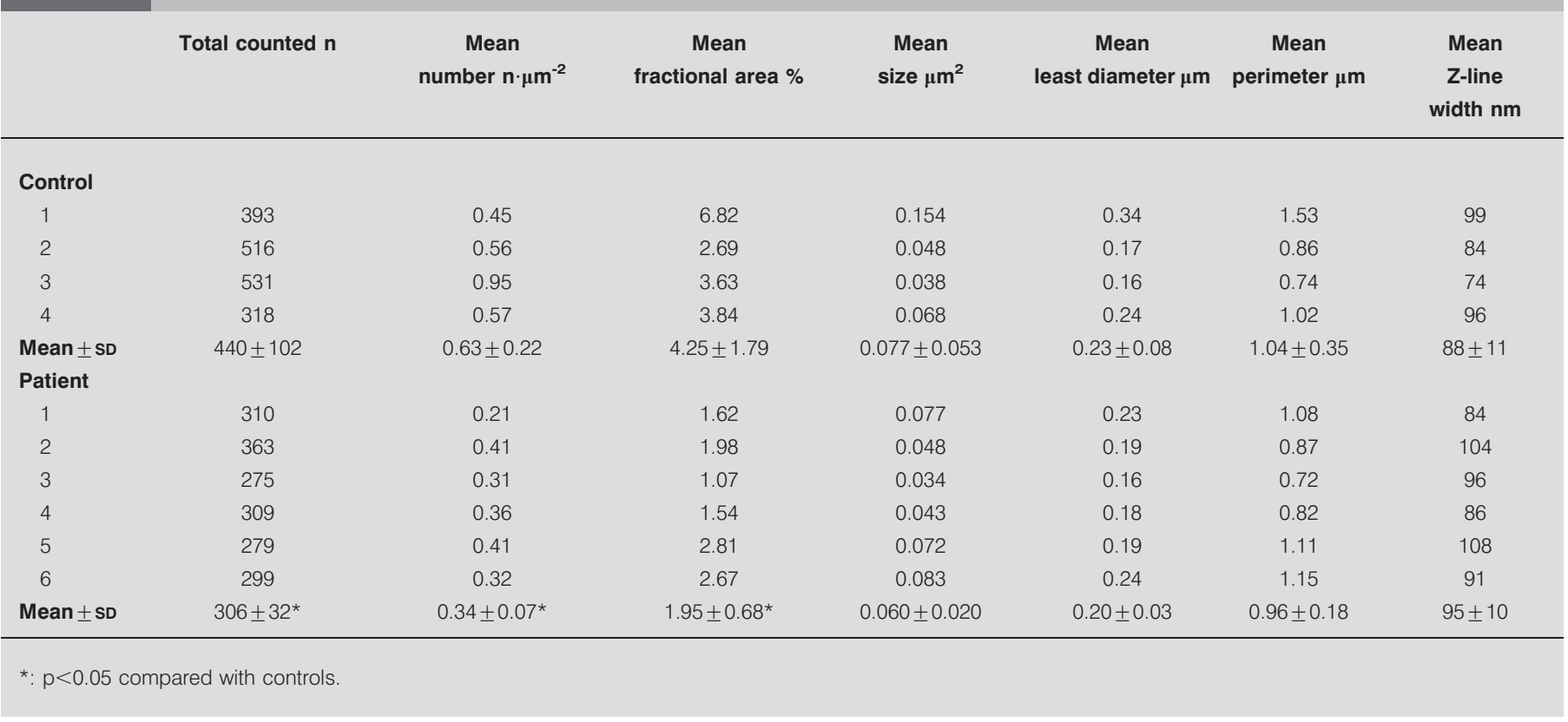

fractional area and not changes in their numbers per se will lead to changes in the mitochondrial capacity of the cell, reflected by altered activities of enzymes hosted by these organelles. The vastus lateralis of COPD patients is indeed characterised by reduced activities of mitochondrial enzymes involved in oxidative energy metabolism, such as citrate synthase and hydroxyacyl-CoA dehydrogenase $[3,4]$. In the current study, both the mitochondrial number and fractional area were reduced in the vastus lateralis of these patients and it is, therefore, very plausible that the observed reduced mitochondrial fractional area contributes to the reported reduced activities of oxidative enzymes. In contrast to the vastus lateralis, tibialis oxidative enzyme activities were found to be normal in COPD [10]. This further strengthens the concept that mitochondrial fractional area determines the oxidative capacity since, despite the fact that the mitochondrial number was reduced in the tibialis of COPD patients, the mitochondrial fractional area was found to be normal in this muscle.

TABLE 3 Mitochondrial parameters in tibialis anterior biopsies

\begin{tabular}{|c|c|c|c|c|c|c|c|}
\hline \multicolumn{8}{|l|}{ Control } \\
\hline 5 & 320 & 0.73 & 3.68 & 0.054 & 0.20 & 0.91 & 116 \\
\hline 6 & 323 & 0.83 & 4.78 & 0.057 & 0.20 & 0.91 & 92 \\
\hline 9 & 296 & 0.92 & 3.65 & 0.040 & 0.18 & 0.78 & 102 \\
\hline 10 & 270 & 0.95 & 4.73 & 0.050 & 0.18 & 0.88 & 93 \\
\hline Mean \pm SD & $305 \pm 20$ & $0.88 \pm 0.13$ & $4.94 \pm 1.85$ & $0.056 \pm 0.013$ & $0.20 \pm 0.02$ & $0.91 \pm 0.1$ & $105 \pm 13$ \\
\hline \multicolumn{8}{|l|}{ Patient } \\
\hline 7 & 448 & 0.65 & 3.93 & 0.058 & 0.20 & 0.94 & 110 \\
\hline 12 & 294 & 0.63 & 2.64 & 0.042 & 0.18 & 0.76 & 89 \\
\hline Mean \pm SD & $351 \pm 108$ & $0.65 \pm 0.05^{\star *}$ & $4.4 \pm 1.22$ & $0.067 \pm 0.016$ & $0.22 \pm 0.02$ & $0.99 \pm 0.13$ & $105 \pm 11$ \\
\hline
\end{tabular}

**: $p<0.01$ compared with controls. 

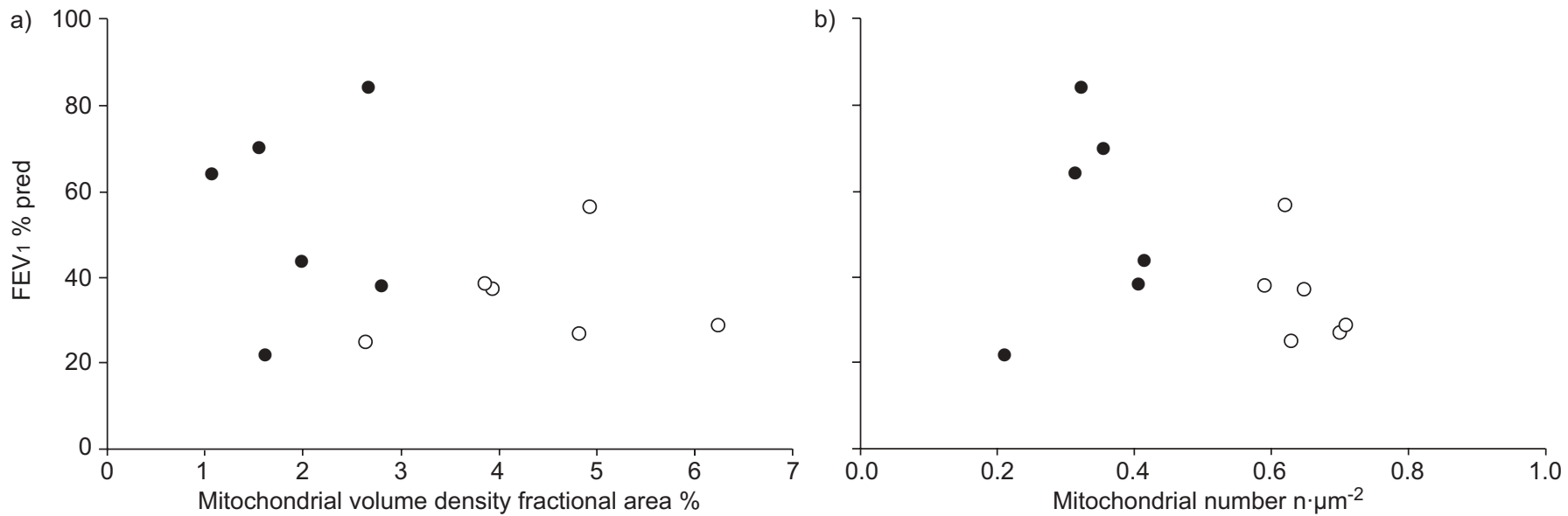

FIGURE 2. Relationship between disease severity and mitochondrial a) volume density and b) number in vastus lateralis $(\bullet)$ and tibialis anterior $(\bigcirc)$ biopsies from patients with chronic obstructive pulmonary disease. No significant correlations were observed. FEV1: forced expiratory volume in one second.

The fact that tibialis mitochondrial fractional area in COPD patients was maintained despite reduced numbers can only be explained by the fact that the tibialis mitochondria tended to be larger in patients. Whether this is a compensatory mechanism and the reasons why this does not occur in the vastus lateralis remain unclear. However, it can be speculated that the vastus lateralis is a muscle primarily involved in locomotion, whereas the tibialis anterior is a constantly-used posture muscle. Concomitant with their function, the tibialis anterior is composed of more fatigue resistant type-I fibres than the vastus lateralis [19]. It can be hypothesised that type-I fibres are more capable of maintaining mitochondrial fractional area than type-II fibres. The key regulator of mitochondrial biogenesis is the peroxisome proliferator-activated receptor- $\gamma$ coactivator-1 $\alpha$ (PGC-1 $\alpha)$. Its expression is highest in type Ifibres [20] and levels are, therefore, probably higher in the tibialis anterior than in the vastus lateralis. One of the target genes of PGC- $1 \alpha$ is mitofusin2, which, as its name indicates,

\begin{tabular}{|c|c|c|}
\hline \multirow[t]{2}{*}{ TABLE 4} & \multicolumn{2}{|c|}{$\begin{array}{l}\text { Scores }(1-5) \text { for the presence of fuel stores in } \\
\text { vastus lateralis biopsies }\end{array}$} \\
\hline & $\begin{array}{l}\text { Glycogen particle } \\
\text { levels }\end{array}$ & $\begin{array}{l}\text { Lipid droplet } \\
\text { levels }\end{array}$ \\
\hline \multicolumn{3}{|l|}{ Control } \\
\hline 1 & 2.5 & 3.4 \\
\hline 2 & 4.3 & 1.7 \\
\hline 3 & 3.6 & 1.3 \\
\hline 4 & 3.7 & 2.6 \\
\hline Mean \pm SD & $3.5 \pm 0.8$ & $2.3 \pm 0.9$ \\
\hline \multicolumn{3}{|l|}{ Patient } \\
\hline 1 & 4.7 & 1.2 \\
\hline 2 & 2.9 & 2.1 \\
\hline 3 & 3.6 & 1.3 \\
\hline 4 & 3.6 & 3.9 \\
\hline 5 & 3.8 & 1.7 \\
\hline 6 & 3.8 & 1.4 \\
\hline Mean \pm SD & $3.7 \pm 0.6$ & $1.9 \pm 1$ \\
\hline
\end{tabular}

plays an important role in the fusion of mitochondria and thus in mitochondrial maintenance [21]. Whether disturbed PGC-1 $\alpha$ and/or mitofusin expression in the vastus lateralis are indeed involved in the loss of mitochondrial fractional area needs further exploration.

The vastus lateralis muscles of patients with COPD are characterised by a I to IIA to IIX fibre type shift [2]. Whether fibre type distribution is also abnormal in the tibialis anterior of COPD patients is presently unknown. Nevertheless, it is theoretically possible that for patients relatively less oxidative type-I fibres from both muscles were included in the analyses, resulting in the observed lower mitochondrial number. However, although it is not possible to identify fibre types from the electron microscopy images directly, it is possible to predict fibre types based on the width of the Z-lines with

\section{TABLE 5 Scores (1-5) for the presence of fuel stores in tibialis anterior biopsies}

\begin{tabular}{|c|c|c|}
\hline & $\begin{array}{c}\text { Glycogen particle } \\
\text { levels }\end{array}$ & $\begin{array}{l}\text { Lipid droplet } \\
\text { levels }\end{array}$ \\
\hline \multicolumn{3}{|l|}{ Control } \\
\hline 5 & 1.9 & 2.4 \\
\hline 6 & 2.9 & 1.4 \\
\hline 7 & 3.5 & 1.5 \\
\hline 8 & 3.2 & 2.0 \\
\hline 9 & 2.9 & 1.8 \\
\hline 10 & 0.7 & 0.5 \\
\hline Mean \pm SD & $2.5 \pm 1$ & $1.6 \pm 0.6$ \\
\hline \multicolumn{3}{|l|}{ Patient } \\
\hline 7 & 2.0 & 1.2 \\
\hline 8 & 3.0 & 1.9 \\
\hline 9 & 1.5 & 1.9 \\
\hline 10 & 3.1 & 1.6 \\
\hline 11 & 3.8 & 1.7 \\
\hline 12 & 3.8 & 1.4 \\
\hline Mean $\pm S D$ & $2.9 \pm 1$ & $1.6 \pm 0.3$ \\
\hline
\end{tabular}


$\sim 83 \%$ accuracy (type-I: $128 \pm 10 \mathrm{~nm}$; type-IIA: $104 \pm 8.5 \mathrm{~nm}$; type-IIX: $88 \pm 9.1 \mathrm{~nm}$ ) with no mismatching between types I and IIX [18]. In the current study, comparing the Z-line widths of the vastus lateralis and the tibialis anterior illustrates that type-I fibre proportions are indeed higher in the latter (although this was not performed in the same subjects). The mean Z-line width in the muscles studied was not different between patients and controls, indicating that any observed differences in mitochondrial density are, at least partly, independent of the fibre type distribution. The present authors also previously showed that oxidative enzyme capacity within a specific fibre type is reduced in vastus lateralis biopsies of patients with COPD [2]. Even despite the fact that patients of the tibialis anterior analysis seemed more severely diseased than those of the vastus lateralis analysis based on FEV1, mitochondrial fractional area is only reduced in the vastus lateralis. Therefore, it can be concluded that the vastus lateralis mitochondrial fractional area is reduced in COPD as compared with healthy subjects and that this by itself contributes to the loss of oxidative capacity in this muscle.

Reduced mitochondrial fractional area along with disturbed energy metabolism may lead to abnormal levels of glycogen and/or lipid stored in the muscle cell. The literature is not consistent regarding glycogen contents in peripheral muscle homogenates of COPD patients [22-24]. Recently, lipid content was examined histochemically in vastus lateralis muscle biopsies of COPD patients and was found to be decreased compared with healthy controls [25]. However, no electron microscopic data of intramuscular fuel stores has been reported for limb muscles of COPD patients. In the current study, no significant differences were found in the quantity scores of lipid and glycogen stores between patients with COPD and healthy subjects, indicating that there was no severe depletion or accumulation of these fuels. It is, however, possible that the power of the current study design is not sufficient to discover more nuanced differences. Furthermore, abnormalities can be expected especially after acute exercise, when these fuels are being used, since the present biopsies were obtained at rest.

There is no golden standard for mitochondrial morphometry but the basic standards are similar to the present authors' approach. Several micrographs were obtained from each sample and from these micrograph parameters, such as mitochondrial count, size and fractional area, are determined by, for example, point-counting using grids or computerised morphometry (as in the current study). Mitochondrial volume densities and Z-line widths reported in these studies are similar to the current findings [17, 18, 26, 27]. Two populations of mitochondria can be distinguished based on the subcellular localisation: 1) intermyofibrillar mitochondria are located between the contractile myofibrils; and 2) subsarcolemmal mitochondria are found adjacent to the sarcolemma, facing the sarcoplasmic site [28]. It is currently not completely clear whether these subtypes have distinct functions within the muscle cell. However, it has been suggested that subsarcolemmal mitochondria are involved in adenosine triphosphate (ATP) production for membrane transport and cytoplasmic reactions, whereas intermyofibrillar mitochondria are more efficient at producing ATP, that is used for muscle contraction [29]. Furthermore, $75 \%$ of the mitochondria are located in the intermyofibrillar space [5]. For these and also some technical reasons, the current study focused on intermyofibrillar mitochondria.

In summary, the present authors showed that the number of mitochondria is reduced in two limb muscles of patients with chronic obstructive pulmonary disease. However, mitochondrial fractional area was only reduced in one muscle, namely the vastus lateralis. As mitochondria are the organelles in which most enzymes involved in oxidative metabolism reside, this loss of mitochondrial fractional area doubtlessly contributes to the impaired oxidative capacity in the vastus lateralis of patients with chronic obstructive pulmonary disease. Therefore, to further unravel the molecular mechanism of impaired muscle oxidative capacity in chronic obstructive pulmonary disease future studies should probably focus on key regulators of mitochondrial maintenance.

\section{REFERENCES}

1 Whittom F, Jobin J, Simard PM, et al. Histochemical and morphological characteristics of the vastus lateralis muscle in patients with chronic obstructive pulmonary disease. Med Sci Sports Exerc 1998; 30: 1467-1474.

2 Gosker HR, van Mameren H, van Dijk PJ, et al. Skeletal muscle fibre type shifting and metabolic profile in patients with COPD. Eur Respir J 2002; 19: 617-626.

3 Maltais F, Simard AA, Simard C, Jobin J, Desgagnes P, LeBlanc P. Oxidative capacity of the skeletal muscle and lactic acid kinetics during exercise in normal subjects and in patients with COPD. Am J Respir Crit Care Med 1996; 153: 288-293.

4 Jakobsson P, Jorfeldt L, Henriksson J. Metabolic enzyme activity in the quadriceps femoris muscle in patients with severe chronic obstructive pulmonary disease. Am J Respir Crit Care Med 1995; 151: 374-377.

5 Elander A, Sjostrom M, Lundgren F, Schersten T, BylundFellenius AC. Biochemical and morphometric properties of mitochondrial populations in human muscle fibres. Clin Sci (Lond) 1985; 69: 153-164.

6 Orozco-Levi M, Gea J, Lloreta JL, et al. Subcellular adaptation of the human diaphragm in chronic obstructive pulmonary disease. Eur Respir J 1999; 13: 371-378.

7 Levine S, Kaiser L, Leferovich J, Tikunov B. Cellular adaptations in the diaphragm in chronic obstructive pulmonary disease. N Engl J Med 1997; 337: 1799-1806.

8 Ribera F, N'Guessan B, Zoll J, et al. Mitochondrial electron transport chain function is enhanced in inspiratory muscles of patients with chronic obstructive pulmonary disease. Am J Respir Crit Care Med 2003; 167: 873-879.

9 Sánchez J, Brunet A, Medrano G, Debesse B, Derenne JP. Metabolic enzymatic activities in the intercostal and serratus muscles and in the latissimus dorsi of middleaged normal men and patients with moderate obstructive pulmonary disease. Eur Respir J 1988; 1: 376-383.

10 Pouw EM, Koerts-de Lang E, Gosker HR, et al. Muscle metabolic status in patients with severe COPD with and without long-term prednisolone. Eur Respir J 2000; 16: 247-252.

11 Fabbri LM, Pauwels RA, Hurd SS, GOLD Scientific Committee, Global strategy for the Diagnosis, Management, and Prevention of Chronic Obstructive Pulmonary 
Disease: Gold Executive Summary updated 2003. COPD 2004; 1: 105-141.

12 Schols AM, Wouters EF, Soeters PB, Westerterp KR. Body composition by bioelectrical-impedance analysis compared with deuterium dilution and skinfold anthropometry in patients with chronic obstructive pulmonary disease. Am J Clin Nutr 1991; 53: 421-424.

13 Quanjer P, Tammeling GJ, Cotes JE, Pedersen OF, Peslin R, Yernault JC. Lung volumes and forced ventilatory flows. Report Working Party Standardization of Lung Function Tests, European Community for Steel and Coal. Official Statement of the European Respiratory Society. Eur Respir J 1993; 6: Suppl. 16, 5-40.

14 Bergstrom L. Muscle electrolytes in man. Determination by neutron activation analysis on needle biopsy specimens. A study on normal subjects, kidney patients, and patients with chronic diarrhea. Scand J Clin Lab Invest 1962; 68: 1-110.

15 Dietrichson P, Coakley J, Smith PE, Griffiths RD, Helliwell TR, Edwards RH. Conchotome and needle percutaneous biopsy of skeletal muscle. I Neurol Neurosurg Psychiatry 1987; 50: 1461-1467.

16 Desplanches D, Hoppeler H, Tuscher L, et al. Muscle tissue adaptations of high-altitude natives to training in chronic hypoxia or acute normoxia. J Appl Physiol 1996; 81: 1946-1951.

17 Poggi P, Marchetti C, Scelsi R. Automatic morphometric analysis of skeletal muscle fibers in the aging man. Anat Rec 1987; 217: 30-34.

18 Sjostrom M, Angquist KA, Bylund AC, Friden J, Gustavsson L, Schersten T. Morphometric analyses of human muscle fiber types. Muscle Nerve 1982; 5: 538-553.

19 Johnson MA, Polgar J, Weightman D, Appleton D. Data on the distribution of fibre types in thirty-six human muscles. An autopsy study. J Neurol Sci 1973; 18: 111-129.

20 Lin J, Wu H, Tarr PT, et al. Transcriptional co-activator PGC-1 alpha drives the formation of slow-twitch muscle fibres. Nature 2002; 418: 797-801.
21 Soriano FX, Liesa M, Bach D, Chan DC, Palacin M, Zorzano A. Evidence for a mitochondrial regulatory pathway defined by peroxisome proliferator-activated receptor- $\gamma$ coactivator- $1 \alpha$, estrogen-related receptor- $\alpha$, and mitofusin 2. Diabetes 2006; 55: 1783-1791.

22 Jakobsson P, Jorfeldt L, Brundin A. Skeletal muscle metabolites and fibre types in patients with advanced chronic obstructive pulmonary disease (COPD), with and without chronic respiratory failure. Eur Respir J 1990; 3: 192-196.

23 Engelen MP, Schols AM, Does JD, Gosker HR, Deutz NE, Wouters EF. Exercise-induced lactate increase in relation to muscle substrates in patients with chronic obstructive pulmonary disease. Am J Respir Crit Care Med 2000; 162: 1697-1704.

24 Pouw EM, Schols AM, van der Vusse GJ, Wouters EF. Elevated inosine monophosphate levels in resting muscle of patients with stable chronic obstructive pulmonary disease. Am J Respir Crit Care Med 1998; 157: 453-457.

25 Russell AP, Somm E, Debigare R, et al. COPD results in a reduction in UCP3 long mRNA and UCP3 protein content in types I and IIa skeletal muscle fibers. J Cardiopulm Rehabil 2004; 24: 332-339.

26 Desplanches D, Hoppeler H, Linossier MT, et al. Effects of training in normoxia and normobaric hypoxia on human muscle ultrastructure. Pflügers Arch 1993; 425: 263-267.

27 Lampert E, Mettauer B, Hoppeler H, Charloux A, Charpentier A, Lonsdorfer J. Structure of skeletal muscle in heart transplant recipients. J Am Coll Cardiol 1996; 28: 980-984.

28 Cogswell AM, Stevens RJ, Hood DA. Properties of skeletal muscle mitochondria isolated from subsarcolemmal and intermyofibrillar regions. Am J Physiol 1993; 264: C383-C389.

29 Mollica MP, Lionetti L, Crescenzo R, et al. Heterogeneous bioenergetic behaviour of subsarcolemmal and intermyofibrillar mitochondria in fed and fasted rats. Cell Mol Life Sci 2006; 63: 358-366. 